RESEARCH ARTICLE

\title{
Morphological Differences Between Imperative and Declarative Pointing: Hand Shape, Arm Extension, and Body Posture
}

\author{
Hélène Cochet ${ }^{1}$, Marianne Jover ${ }^{2}$, Lucie Oger ${ }^{2}$, Jacques Vauclair ${ }^{2}$ \\ ${ }^{1}$ Laboratory "Cognition, Communication and Development" (Octogone - ECCD, EA 4156) University of Toulouse II, \\ Department of Psychology, Toulouse, France. ${ }^{2}$ Aix Marseille Université, PsyCLE EA 3273, 13621, Aix en Provence, France.
}

\begin{abstract}
The authors used frame-by-frame video analyses to describe the features of imperative and declarative pointing gestures produced by young children, in comparison to reaching actions. First, the results showed that imperative pointing shared common features with reaching actions (hand shape, arm extension), but body posture observed in reaching differed from the one observed in pointing, both in imperative and declarative contexts. Second, hand shape was influenced by precision constraints: imperative gestures shifted from whole-hand pointing to index-finger pointing when the target was surrounded by distractors. This study is the first of its kind to highlight the effect of several variables on morphological features of pointing using quantitative measures and may provide insights into the nature of imperative and declarative pointing.
\end{abstract}

Keywords: arm extension, body posture, gestural communication, hand shape, imperative and declarative pointing, reaching

$\mathbf{T}$ he development of gestural communication has been the topic of many studies over the previous 40 years. Regarded as the "royal road to language" (Butterworth, 2003), pointing has been the main focus of researchers (e.g., Colonnesi, Stams, Koster, \& Noom, 2010; Rowe \& GoldinMeadow, 2009), and yet the physical features of this gesture have never been described precisely. This is all the more surprising as the characteristics of noncommunicative manual activities, such as reach-to-grasp movements, have been largely investigated (e.g., Fagard, 2000; Sacrey, Karl, \& Whishaw, 2012; Seegelke, Hughes, \& Schack, 2011). The objective of the present study was, therefore, to (a) provide quantitative analyses of morphological features associated with pointing gestures in young children (hand shape, arm extension, and body posture) and (b) determine to what extent these features vary with variables such as the function served by pointing and the ambiguity of the context.

Children start to produce pointing gestures before they reach 1 year of age (e.g., Butterworth \& Morissette, 1996; Camaioni, Perucchini, Bellagamba, \& Colonnesi, 2004), for imperative purposes when they want the adult to give them a specific object or to act on that object, but also in declarative contexts when they seek to share some interest with the adult about an external object or event (e.g., Camaioni, 1997). Some researchers have argued that declarative pointing, at least in early stages, was actually produced to direct the other's attention toward the self and obtain a positive emotional reaction (Moore \& Corkum, 1994). However, experimental studies tend to show that declarative pointing is not simply an instrumental gesture and involves the understanding of the adult's attention and intention (e.g., Liszkowski, Carpenter, Henning, Striano, \& Tomasello, 2004).
The observation of several differences between imperative and declarative pointing also suggests that different processes come into play for the emergence of these gestures. First, longitudinal studies have shown that imperative and declarative pointing do not emerge at the same period in the course of development (Camaioni et al., 2004; Carpenter, Nagell, \& Tomasello, 1998). Second, vocalizations accompany more frequently declarative pointing than imperative pointing (Cochet \& Vauclair, 2010a), which may reflect a stronger role of declarative pointing in language acquisition. Moreover, declarative pointing is mostly produced with the index finger extended, whereas imperative pointing is usually characterized by whole-hand gestures (Cochet \& Vauclair, 2010b; Franco \& Butterworth, 1996; Liszkowski $\&$ Tomasello, 2011). This difference in hand shape might be explained by distinct origins of the two types of pointing and suggests that imperative pointing may emerge from unimanual reaching (e.g., Cochet, Jover, \& Vauclair, 2011). Both activities share indeed a common form (an open hand) and a common goal (obtaining an object), one hypothesis being that reaching action gradually acquires a communicative function through a process of ontogenetic ritualization (Bruner, 1978; Tomasello \& Call, 1997). By contrast, declarative pointing has been argued to develop in association with some social and cognitive abilities, in particular imitation and cooperation skills (e.g., Liszkowski, Carpenter, \& Tomasello, 2008).

However, to our knowledge, the difference between imperative and declarative pointing has never been illustrated with quantitative measures that would allow us to go beyond the index-finger/whole-hand distinction and consider intermediate hand shapes. Such quantitative measures may also allow researchers to use more precise definitions for coding gestures in developmental studies, and especially to clarify the difference between arm-point (or palm-point) gestures and reaching gestures (or ritualized reaches; e.g., Franco, Perucchini, \& March, 2009; Iverson \& Goldin-Meadow, 2005). In addition, the study of body posture and arm extension may provide further arguments to support-or not - the previously mentioned hypotheses regarding the origins of imperative and declarative pointing. Children may be more likely to lean toward an object or fully extend the arm (e.g., Kaminski, Bock, \& Gentile, 1995) when they want to obtain

Correspondence address: Hélène Cochet, Laboratory "Cognition, Communication and Development" (Octogone - ECCD, EA 4156) University of Toulouse II, Department of Psychology, 5 allées Antonio Machado, 31058 Toulouse Cedex 9 - France. e-mail: helene.cochet@univ-tlse2.fr 
that object than when they show it to the adult for declarative purposes.

The production of pointing gestures can also be influenced by external variables. First, the target location has been shown to influence hand choice for pointing: children tend to use the hand that is ipsilateral to the hemivisual field of the referent (e.g., Esseily, Jacquet, \& Fagard, 2011). Second, the salience of the target has an effect on the frequency of pointing: objects in motion or with auditory displays are more likely to elicit pointing gestures than static and silent objects (e.g., Butterworth, Franco, McKenzie, Graupner, \& Todd, 2002). Moreover, children seem able to adapt their communicative behaviors to some referential ambiguity. For example, children have been reported, from the end of the third year, to complement their pointing gestures with a verbal descriptor more frequently when the potential referents are side by side than when they are farther apart (O'Neill \& Topolovec, 2001). Similarly, we may assume that children use all the more precise deictic gestures as the context is more confusing. A whole-hand point is effective when there is little ambiguity to identify the target, for example when there is only one object in the proximal environment of communicative partners. The presence of several objects around the target may on the contrary require a more precise gesture, namely a gesture with the index finger extended and the other fingers tightly curled. However, this hypothesis still has to be tested. This is the reason why we sought to investigate in this study the effect of precision constraints on the features of pointing, especially on hand shape, in children between 3 and 4 years of age.

The parameters associated with gestures production can be measured accurately via the use of external markers placed for example on the fingertips; however, we excluded this possibility in the present study to rule out any bias in the way children may use their hands. In effect, children are likely to focus their attention on such markers, which may influence the form of their gestures. We chose instead to film each interaction and use a program to analyze frame by frame the displacement of body landmarks such as the fingertip or the nose. Our first objective was to provide quantitative measures of imperative and declarative pointing in order to compare hand shape, arm extension and body posture associated with these two functions. The measure of the same parameters for reaching may allow us to determine precisely common features and divergences between pointing and reaching. Experimental tasks were performed by children between 3 and 4 years of age in order to focus on a relatively homogenous phase for the development of gestural communication (i.e., more homogenous than for the period of lexical acquisition and for the transition to two-word utterances; e.g., Özçaliskan \& Goldin-Meadow, 2009). Pointing behaviors were thus more likely to be stable in all children. We expected to observe a wide-open hand for reaching actions and imperative pointing and a fully closed hand for declarative pointing. The body contribution, including body posture and arm extension, may be stronger for reaching actions and imperative pointing than for declarative pointing: we hypothesized to observe a smaller distance between the children's bodies and the target and a greater arm extension for reaching and imperative pointing.

Our second objective was to examine the effects of precision constraints on the morphology of pointing gestures. We set up two different conditions for both imperative and declarative pointing, opposing an obvious condition in which the target was presented alone to a more ambiguous condition in which the target was presented at the same location, but surrounded by two distractors. We expected the shape of imperative gestures to shift from whole-hand pointing to index-finger pointing with the ambiguity of the situation. We expected to observe a similar change for declarative gestures, though to a lesser degree as this gesture has been reported to be characterized by index-finger pointing even in conditions with only one apparent target (Cochet \& Vauclair, 2010b).

\section{Method}

\section{Participants}

Fourteen French native-speaking children (five boys and nine girls), aged between 41 and 51 months old ( $M$ age $=45.6$ months; $S D=3.5$ months), were tested individually in a daycare center. We chose this age range as pointing skills would be mastered and stable in all children, therefore allowing for reliable comparisons across situations. We also wanted to make sure that all children would be fully aware of the potential referential inefficacy of their pointing gestures in the ambiguous condition (O’Neill \& Topolovec, 2001), and could thus adapt their gestures. Written informed consent forms were obtained from the parents before the start of the study. The experimenter had spent one day in the day-care center prior to the experiments so that the children could familiarize with her during free play time.

\section{Material}

For the three tasks, we used cubes of the same dimensions (side length of $5.5 \mathrm{~cm}$ ), with pictures stuck on them (pictures were different for the five trials of each task). For the imperative pointing task, we used five series of three cubes with a small hole in the center, so that they could fit on a wooden stick, which was maintained on a rigid support. The first set represented the head, trunk, and legs of a person, and the other sets consisted of three identical cubes (cubes with the picture of a ball, cubes with the picture of a candy, cubes covered with feathers, and cubes covered with glitter). For the declarative pointing task and the reaching task, we used five cubes with different pictures (e.g., a dog, a car, a bird). For the declarative pointing task, we also used an opaque sheet of paper (A4 format: $21 \times 29.7 \mathrm{~cm}$ ) as a cover to hide the cubes (see hereafter). The objects used as distractors in the pointing tasks (condition B) were simple wooden cubes without any pictures or distinctive features. 


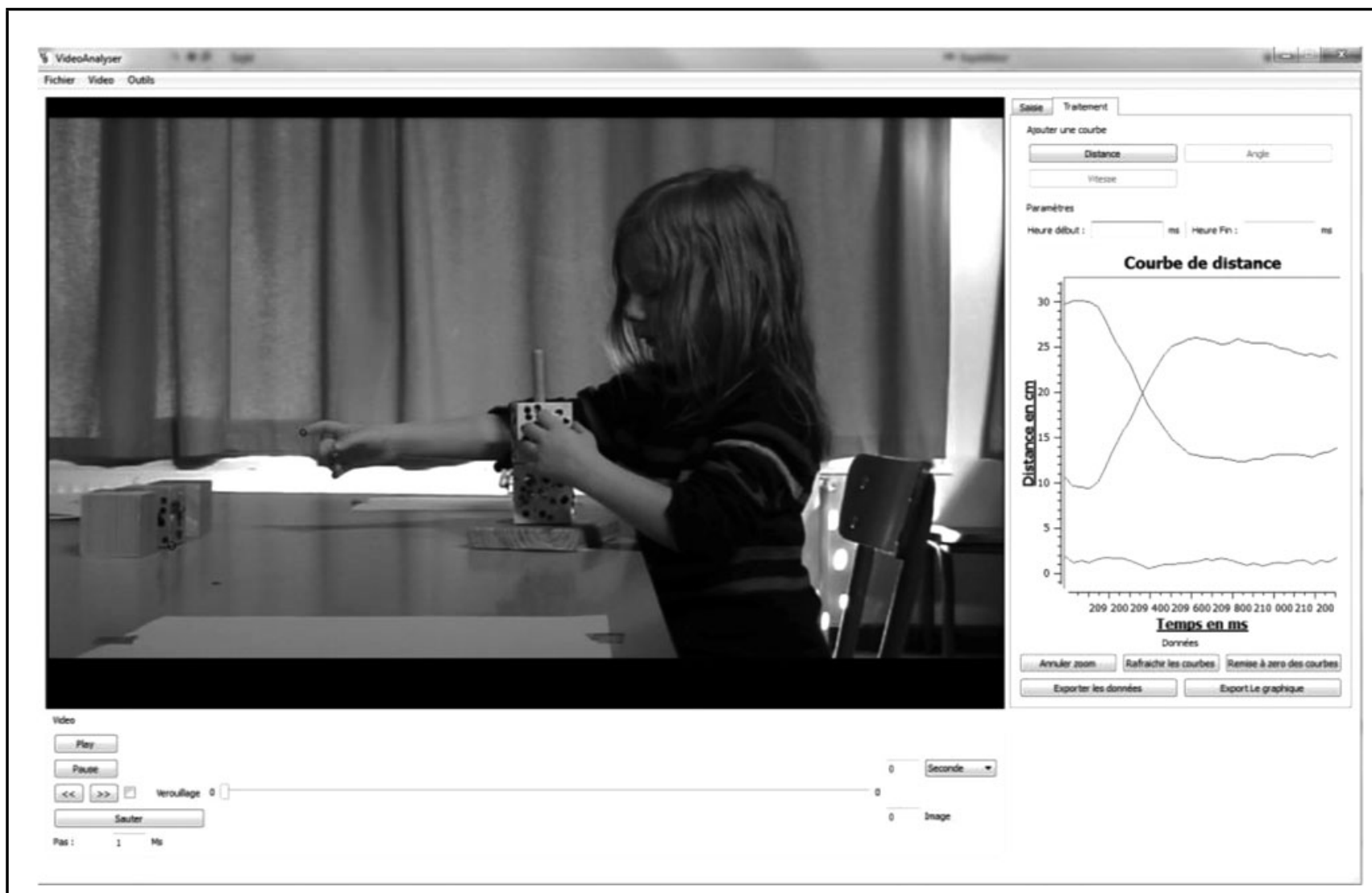

FIGURE 1. Video Analyser interface. Circle-shaped markers were positioned frame by frame on the index finger and middle finger extremities (respectively $I$ and $M$ ), on the lower left corner of the target cube $(R)$ and on the child's nose $(N)$. Graphs illustrated the changes during the movement (at a frequency of $25 \mathrm{~Hz}$ ) in the $I M$ distance (hand shape), the $I N$ distance (arm extension), and the $N R$ distance (body posture).

All sessions were video recorded. Two cameras were positioned opposite to each other on the right and the left of the child, $90 \mathrm{~cm}$ away from the table. Body landmarks displacements in pointing and reaching were measured with Video Analyser, a program developed in our lab (Mare, 2010). This program allows the conversion of markers, manually positioned frame by frame, into $x$ and $y$ coordinates, thus providing distances of interest as well as graphic illustrations of the predefined variables (see Figure 1 for an overview of the interface and the Measures section for more details).

\section{Procedure}

Children were at a table, opposite the experimenter in a room apart from the other children. Child-size furniture was used. A caregiver from the day-care center was present in the room if the child seemed more at ease that way, but he or she was asked not to interfere with the experiment. After a short warm-up period, each child was tested individually for three tasks: the unimanual reaching task, the imperative pointing task, and the declarative pointing task. The experimental designs used to induce imperative and declarative pointing were based on tasks proposed in previous studies (e.g., Cochet et al., 2011; Liszkowski, Schäfer, Carpenter, $\&$ Tomasello, 2009; for a description of indicative and request situations, see also Blake, O'Rourke, \& Borzellino, 1994). Both obvious and ambiguous conditions (conditions A and B) were performed for the pointing tasks, namely with only the target object in front of the child or with two adjacent distractors, respectively. The reaching task was administered first, and the order of imperative and declarative pointing tasks was alternated across participants. This order of presentation allowed us to facilitate the interactions with the children (pointing tasks usually require a higher level of familiarity with the adult compared to reaching tasks), and to prevent any confusion between noncommunicative movements and communicative gestures. The conditions $\mathrm{A}$ and $\mathrm{B}$ of the pointing tasks were randomly mixed, but we excluded the possibility of having the same condition more than two consecutive times. There were five trials per condition, leading to 10 trials for each pointing task and to a total of 25 trials. In order to clearly distinguish the trials and start from the same initial position, the experimenter made sure that the children had repositioned their hands on two circles 
drawn on the table between each trial. The target cube used in all tasks and all conditions was always positioned on the table at the exact same place, at a distance equivalent to $150 \%$ of the child's arm length (approximately $40 \mathrm{~cm}$ ), so that children could not reach the cubes from the seated position.

\section{Unimanual Reaching Task}

One of the cubes was positioned on the table in front of the child in a midline position, out of reach. The experimenter then asked the child whether he or she could get the cube while staying seated on the chair in order to elicit reaching movements.

\section{Imperative Pointing Task}

In the obvious condition (A), the experimenter first demonstrated to the child the way the cubes could be piled up together on the wooden stick. She then gave the stick to the child with only two piled-up cubes, and invited him or her to reproduce the same game. The third cube was positioned on the table in a midline position, out of the child's reach. If the child produced a pointing gesture, the adult gave him or her the missing cube. If the child did not produce any gesture within a 10-s delay, the experimenter tried to elicit a gesture by looking at the cube and saying with enthusiasm that there was still some place for another cube on the stick. We used the same procedure in the ambiguous condition (B), but the third cube was positioned on the table between two distractors, that is between two simple wooden cubes without any hole. The distance between each cube was $2 \mathrm{~cm}$ and the target cube was at the exact same place as in condition A. If the child pointed toward one of the distractors, the experimenter gave it to him or her, but the trial was not considered for analyses.

\section{Declarative Pointing Task}

The experimenter presented this task as a game, the goal of which was to discover nice pictures. In the obvious condition (A), the opaque sheet was maintained on the table so that the child could not see the cube as it was positioned by the experimenter in a midline position, out of the child's reach. The experimenter then told the child that she was about to remove the sheet and that he or she could see some pictures. As soon as the sheet was removed, she asked the child where the target picture was (e.g., the dog). If the child produced a pointing gesture, the experimenter showed some enthusiasm while commenting on the picture and hid another cube with the sheet for the next trial (the child was not given the target cube). The same procedure was used in the ambiguous condition (B), except that the target cube was placed between two simple cubes, namely the same distractors as those used in the imperative task. The distance between each cube was $2 \mathrm{~cm}$ and the target cube was at the exact same place as in condition A.

\section{Measures}

The videos obtained were analyzed frame by frame (at a frequency of $25 \mathrm{~Hz}$ ) with Video Analyser (Mare, 2010). This program was developed in our laboratory to describe the morphological features of movement by providing the $x, y$ coordinates of several markers in reference to the sagittal plane of the participant. In addition to a fixed point of reference $(R$, which was the lower left corner of the cube placed on the table), we positioned three circle-shaped markers on each frame corresponding to a pointing gesture or a reaching movement. We chose as markers the extremity of the children's nose $(N)$, the index extremity $(I)$, and the middle finger extremity $(M)$. We had the possibility to zoom on the frame and to adjust the size of the markers for more accuracy (see Figure 1). The program provided the distances (in $\mathrm{cm}$ ) between these different markers as well as the distance between the markers and the reference point. All distances were calculated with respect to the sagittal plane.

The $I M$ distance was used as a measure of hand shape, increasing on a continuum from whole-hand pointing and reaching to index-finger pointing. We calculated the mean distance for each trial. The $I N$ distance reflected the degree of arm extension, this distance being maximal when the arm is fully stretched. We selected the maximal distance within each trial as the most relevant measure. The $N R$ distance was used as an indicator of the child's posture because this distance decreases as the body leans toward the cube. We selected the minimal distance within each trial as the most relevant measure. The analysis of each movement (pointing and reaching) began with the frame preceding the first visible movement of the child's hand and ended when the child moved his/her hand back (or in a few cases when he or she slightly stood up from the chair and touched the object).

\section{Results}

For the three distances of interest, we first calculated for each child the mean values from the five trials performed in each task and each condition. Second, we calculated the mean distances obtained at the group level (see Table 1). Given that the data did not follow a normal distribution, we performed nonparametric statistical tests to analyze (a) the relationship between pointing gestures and reaching movement, (b) the relationship between the obvious (A) and the ambiguous (B) conditions, and (c) the interaction between the function of pointing (imperative vs. declarative) and the condition (A vs. B). In each of these three sections, analyses included both comparisons between tasks or between conditions (using the Friedman test and Wilcoxon test) and Spearman rank correlations. The significance threshold was set at $p<.05$, but Bonferroni corrections were applied to adjust the level of significance when multiple comparisons were performed. 
TABLE 1. Mean Distances (in cm) Observed for Reaching Actions, Imperative Pointing, and Declarative Pointing in the Obvious (A) and Ambiguous (B) Conditions for the 14 Participants

\begin{tabular}{|c|c|c|c|c|c|c|c|c|c|c|}
\hline & & & \multicolumn{4}{|c|}{ Imperative pointing } & \multicolumn{4}{|c|}{ Declarative pointing } \\
\hline & \multicolumn{2}{|c|}{ Reaching action } & \multicolumn{2}{|c|}{ A } & \multicolumn{2}{|c|}{ B } & \multicolumn{2}{|c|}{ A } & \multicolumn{2}{|c|}{$\mathrm{B}$} \\
\hline & $M$ & $S D$ & $M$ & $S D$ & $M$ & $S D$ & $M$ & $S D$ & $M$ & $S D$ \\
\hline Hand shape: IM distance (cm) & 1.74 & 0.79 & 2.15 & 0.70 & 2.57 & 1.00 & 3.19 & 1.00 & 3.29 & 1.20 \\
\hline Arm extension: IN distance $(\mathrm{cm})$ & 22.90 & 4.19 & 24.00 & 4.49 & 24.90 & 5.12 & 25.20 & 4.66 & 24.50 & 4.94 \\
\hline Posture: NR distance $(\mathrm{cm})$ & 23.10 & 4.20 & 28.10 & 4.86 & 28.20 & 5.10 & 28.10 & 6.14 & 26.80 & 6.14 \\
\hline
\end{tabular}

\section{Comparison Between Reaching Actions and Pointing Gestures (Condition A)}

We compared reaching actions with pointing gestures produced in condition A to analyze situations with only one object present on the table. Friedman tests revealed a significant effect of the type of movement on the $I M$ distance (hand shape), $\chi^{2}(2, N=14)=23.6, p<.001$ and on the $N R$ distance (body posture), $\chi^{2}(2, N=14)=18.6, p<$ .001 . The Friedman test tended to be significant for arm extension, $\chi^{2}(2, N=14)=8.29, p=.082$. Two-by-two comparisons using Wilcoxon tests showed that the $I M$ distance (hand shape) was greater for declarative pointing than for reaching $(Z=2.92, p<.01)$, but there was no significant difference between imperative pointing and reaching $(Z=$ $1.60, p=n s$, see Figure 2). The comparison between imperative and declarative pointing is detailed subsequently, in the interaction paragraph.

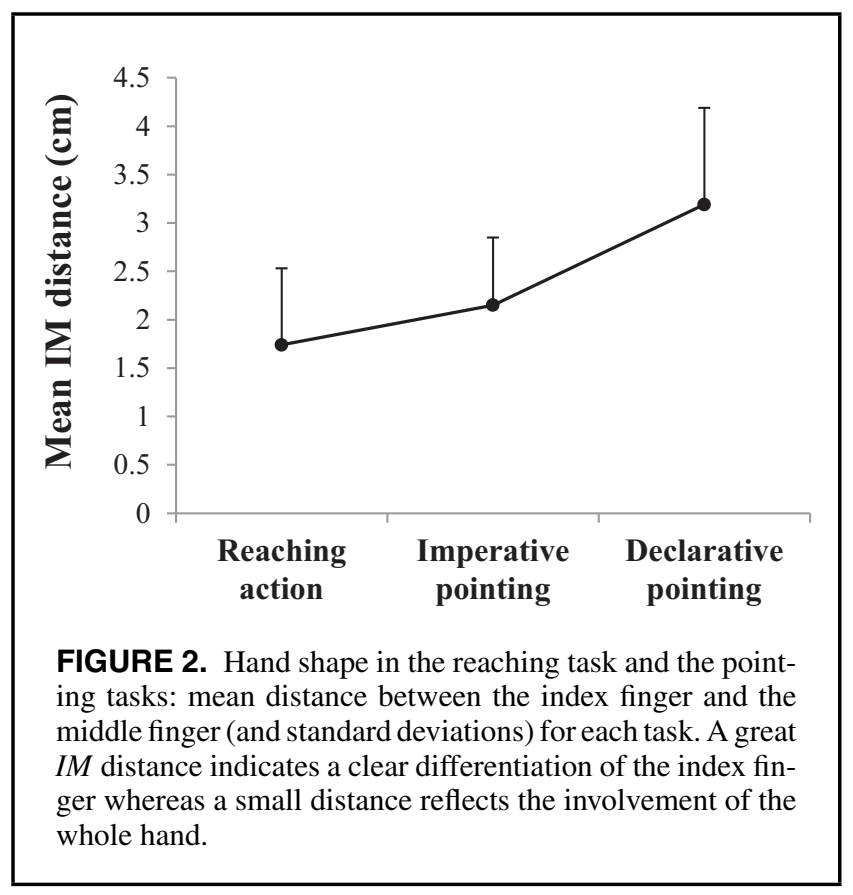

The IN distance (arm extension) was greater for declarative pointing than for reaching $(Z=2.29, p<.05)$, but there was no significant difference between imperative pointing and reaching $(Z=1.29, p=n s)$. The $N R$ distance (body posture) was greater for pointing than for reaching, in imperative context $(Z=3.11, p<.01)$ and declarative context $(Z=$ $2.79, p<.01$; see Figure 3 ).

Spearman rank correlations revealed a significant relationship for $I M$ distance (hand shape) between reaching actions and imperative pointing $(R=.54, p<.05)$, but not between reaching actions and declarative pointing $(R=.099$, $p=n s)$. Similarly, there was a significant correlation in $N R$ distance (body posture) between reaching actions and imperative pointing $(R=.60, p<.05)$, but not between reaching actions and declarative pointing $(R=.21, p=n s)$. The $I N$ distance (arm extension) in reaching actions was significantly correlated both to the $I N$ distance in imperative pointing $(R=.64, p<.05)$ and in declarative pointing $(R=.70$, $p<.01)$.

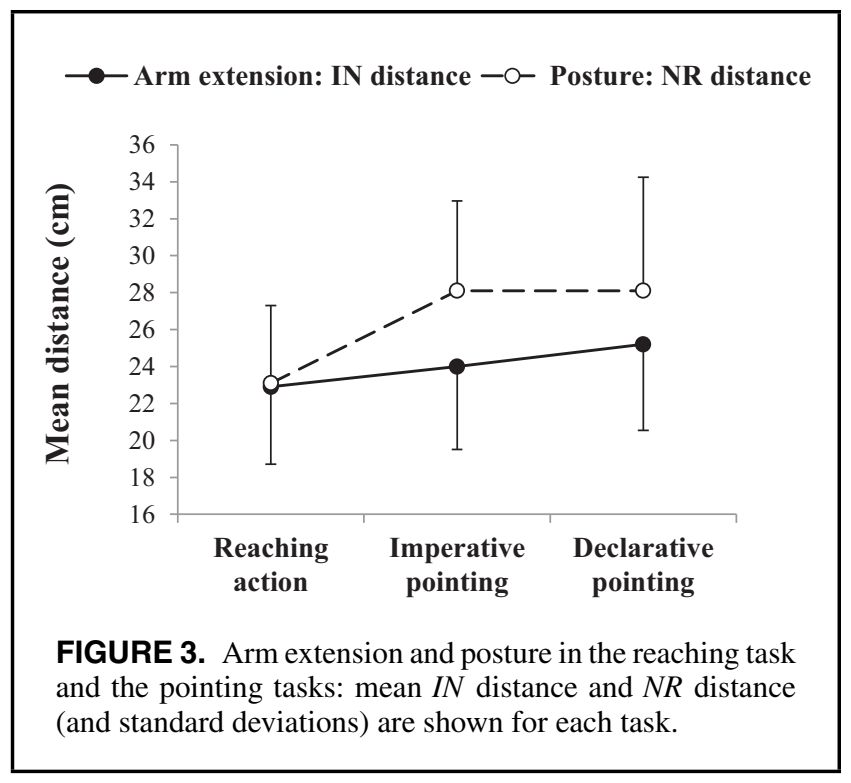




\section{Comparison of the Obvious (A) and Ambiguous (B) Conditions in the Two Tasks}

\section{For Imperative Pointing}

Wilcoxon tests showed that the $I M$ distance (hand shape) was greater in condition $\mathrm{B}$ than in condition $\mathrm{A}(Z=2.17$, $p<.05$ ), but there was no significant difference between both conditions neither in the IN distance (arm extension; $Z=1.16, p=n s$ ) nor in the $N R$ distance (body posture; $Z=0.032, p=n s)$.

\section{For Declarative Pointing}

There was no significant difference between the two conditions in neither the $I M$ distance (hand shape; $Z=0.16$, $p=n s$ ) nor the $I N$ distance (arm extension; $Z=1.66, p=$ $n s)$. The $N R$ distance (body posture) was greater in condition A than in condition $\mathrm{B}(Z=2.61, p<.01)$.

\section{Interaction Between the Function of Pointing and the Condition}

The comparison between imperative and declarative pointing showed that the $I M$ distance (hand shape) was greater in declarative pointing than in imperative pointing in condition A $(Z=2.73, p<.01$; see Figure 2$)$, but there was no significant difference in condition $\mathrm{B}(Z=1.66, p=n s$; see Figure 4). There was no significant difference in $I N$ distance (arm extension) between imperative pointing and declarative pointing in neither condition $\mathrm{A}(Z=1.29, p=n s)$ nor condition $\mathrm{B}(Z=1.04, p=n s)$. There was no significant difference in $N R$ distance (body posture) between imperative pointing and declarative pointing in neither condition A $(Z=0.47$, $p=n s)$ nor condition $\mathrm{B}(Z=1.41, p=n s)$.

Finally, correlational analyses reported in Table 2 revealed significant relationships between the conditions A and B for

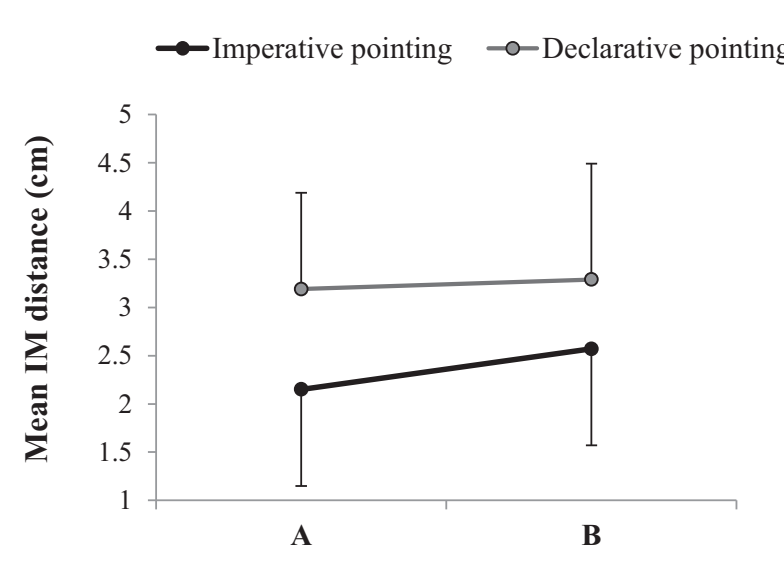

FIGURE 4. Hand shape: mean $I M$ distances (and standard deviations) for imperative and declarative pointing are shown for the obvious condition (A, one target alone) and the ambiguous condition (B, one target with 2 distractors).
$I M$ distance (hand shape) and NR distance (body posture), in both imperative and declarative pointing, but not between the two functions of pointing. By contrast, we observed significant correlations between the different functions and conditions of pointing for $I N$ distance (arm extension).

\section{Discussion}

In this study, we have set up different experimental situations to elicit imperative and declarative pointing gestures in young children. Using frame-by-frame video analyses with a specific software, we have provided a quantitative description of several structural features associated with pointing gestures, compared to unimanual reaching. One of our objectives was to compare hand shape, arm extension, and body posture to shed some light on the continuities and divergences between imperative and declarative pointing (e.g., Cochet \& Vauclair, 2010a; Lock, Young, Service, \& Chandler, 1990). We also manipulated the level of precision constraints to investigate the influence of these constraints on the morphology of pointing. We will discuss these two questions successively.

The measure of the mean distance between the extremity of the index and that of the middle finger (IM) has highlighted similar hand shape in imperative pointing and reaching, whereas the form of declarative pointing was significantly different from hand shape associated with reaching actions and imperative pointing. In addition, correlational results have revealed (a) a significant relationship between imperative pointing and reaching actions, but not between declarative pointing and reaching actions and (b) the absence of any significant correlation in hand shape between imperative pointing and declarative pointing. These results are in line with our hypothesis and with previous studies showing that imperative pointing is characterized by whole-hand gestures while declarative pointing is mostly associated with index-finger gestures (Cochet \& Vauclair, 2010b; Franco \& Butterworth, 1996; Liszkowski \& Tomasello, 2011). The present study allowed us to quantify these differences and to focus also on other features: the body posture (reflected by the distance between the object and the child's nose) observed for reaching actions was different from that observed for pointing gestures, both in imperative and declarative contexts. As expected, reaching actions were characterized by a greater body contribution than pointing gestures (i.e., children leaned closer to the cube). The larger distance observed in the pointing tasks further highlights the communicative nature of these gestures: children intentionally addressed the adult to convey either an imperative or declarative message, whereas they sought to achieve their goals through direct motor action on the object in the reaching task. However, the posture adopted in reaching actions was significantly correlated with the posture observed in imperative pointing, but not with that observed in declarative pointing.

Moreover, arm extension (assessed via the distance between the nose and the index finger) was found to be equivalent in imperative pointing and reaching actions, but was 
TABLE 2. Spearman Rank Correlations Between Imperative and Declarative Pointing Gestures in the Conditions A and B ( $n=14)$ for the IM Distance (a), the NR Distance (b), and the IN Distance (c)

\begin{tabular}{|c|c|c|c|c|}
\hline & & $\begin{array}{l}\text { Imperative } \\
\text { pointing B }\end{array}$ & $\begin{array}{l}\text { Declarative } \\
\text { pointing A }\end{array}$ & $\begin{array}{l}\text { Declarative } \\
\text { pointing B }\end{array}$ \\
\hline \multirow[t]{3}{*}{ a. $I M$ distance (hand shape) } & Imperative pointing $\mathrm{A}$ & $.65^{*}$ & .042 & .37 \\
\hline & Imperative pointing $\mathrm{B}$ & & .068 & .31 \\
\hline & Declarative pointing A & & & $.76^{* *}$ \\
\hline \multirow[t]{3}{*}{ b. $N R$ distance (body posture) } & Imperative pointing $\mathrm{A}$ & $.95^{* * *}$ & .12 & .15 \\
\hline & Imperative pointing $\mathrm{B}$ & & .06 & .13 \\
\hline & Declarative pointing A & & & $.98^{* * *}$ \\
\hline \multirow[t]{3}{*}{ c. IN distance (arm extension) } & Imperative pointing $\mathrm{A}$ & $.78^{* * *}$ & $.68^{* *}$ & $.69^{* *}$ \\
\hline & Imperative pointing $B$ & & $.64^{*}$ & $.63^{*}$ \\
\hline & Declarative pointing A & & & $.96^{* * *}$ \\
\hline
\end{tabular}

${ }^{*} p<.05 .{ }^{* *} p<.01 .{ }^{* * *} p<.001$

greater in declarative pointing than in reaching. However, there was no significant difference between imperative pointing and declarative pointing. We thus did not observe the greatest arm extension for reaching actions, but this might be due to the fact that, as mentioned previously, children leaned closer toward the cube in this task. A greater contribution of the trunk, associated with a less important arm extension, has also been reported in adults in a comparable task of objects touching (Kaminski et al., 1995). Moreover, in the present study, arm extension in reaching actions was significantly correlated with arm extension in imperative pointing and declarative pointing, indicating that children who only slightly extended their arms in one task also slightly extended their arms in another task.

Overall, the analyses of hand shape, arm extension, and body posture have emphasized (a) the distinction between communicative gestures and noncommunicative activities when body posture was compared and (b) the distinction between imperative and declarative pointing when we focus on hand shape and on some correlational results. Both reaching and pointing are intentional and object-directed actions, but only pointing has a communicative meaning. This difference, manifesting itself in the characteristics of children's manual actions, may imply some representation of the gesturer's intentions: children are more likely to attribute knowledge to an ignorant experimenter when the latter points toward an object than when he or she grasps the same object (Palmquist \& Jaswal, 2012). Brinck (2001) also argued that imperative and declarative gestures constitute one and the same way of communicating, with the underlying hypothesis that the latter directly evolve from the former.

In this respect, one of the aims of the present study was to provide some clues to the nature of pointing gestures based on measures of their morphological features. Although it should be kept in mind that distinct morphological features do not automatically reflect distinct origins of gestures, and vice versa, the whole set of results in the present study may suggest that different processes come into play in the emergence of imperative and declarative pointing. First, imperative pointing was found to be similar to reaching actions for two of the three features studied (hand shape and arm extension). Second, hand shape and body posture observed in imperative pointing and reaching were significantly correlated, whereas the same variables in declarative pointing were not correlated with those associated with reaching or imperative pointing. Differences between the two types of gestures in the frequency of accompanying vocalizations, gaze behavior, and hand preference have been described in previous studies (e.g., Cochet et al., 2011; Cochet \& Vauclair, 2010a, 2010b; Franco \& Butterworth, 1996). In addition, longitudinal observations have revealed significant relationships between the production of comment gestures, including declarative pointing, and later social and communicative abilities, whereas the production of imperative gestures was not correlated, or negatively correlated to these abilities (Blake, Vitale, Osborne, \& Olshansky, 2005; Camaioni et al., 2004).

Altogether, these findings might therefore suggest that imperative pointing originates from reach-to-grasp actions, which, because caregivers are likely to infer the child's intention from these actions, would gradually acquire a communicative meaning (e.g., Vygotsky, 1988). Such interactions, referred to as ontogenetic ritualizations (Tomasello \& Call, 1997), may allow infants to develop social skills involving the understanding of others' attention and intentions (e.g., Liszkowski et al., 2008; Moll \& Tomasello, 2007). The emergence of declarative pointing, which has been reported to occur after that of imperative pointing (Camaioni et al., 2004), might rely on these early social-cognitive abilities (e.g., Matthews, Behne, Lieven, \& Tomasello, 2012).

It has also been argued that pointing develops as an extension of fingertip exploration through gradual social shaping (e.g., Masataka, 2003), but this hypothesis concerns mainly what has been regarded as canonical pointing gesture, namely gestures with the extended index finger and the other fingers 
curled (e.g., Carpendale \& Carpendale, 2010). However, our results suggest that restricting the question of the origins of pointing to index-finger pointing is tantamount to studying mostly, if not exclusively, declarative pointing. Although imperative and declarative pointing are both communicative acts, we argue here that it may be necessary to distinguish between these different functions to examine gestural communication in children. Thus, although it is difficult to empirically prove hypotheses about the origins of gestures, focusing on different functions of pointing may allow researchers to go beyond the distinction between social versus nonsocial origins of pointing (e.g., Bruner, 1983; Carpendale \& Carpendale, 2010; Leung \& Rheingold, 1981). There may not be a simple causal relationship between the development of pointing gestures and that of social and cognitive skills. Abilities of shared attention, which manifest themselves before infants produce any gesture, for example through gaze following and turn-taking behaviors, first offer a foundation for the development of communicative skills (e.g., Carpenter, Nagell, \& Tomasello, 1998; Heimann et al., 2006; Kristen, Sodian, Thoermer, \& Perst, 2011). Then, from a noncommunicative action, imperative pointing may initiate the development of more complex social-cognitive competencies, linked to the representation of adults' intentions. Declarative pointing might subsequently emerge out of these competencies and may also gradually enhance further the acquisition of intention-reading skills (e.g., Tomasello, 2008). However, although our results are consistent with the hypothesis of distinct origins of imperative and declarative pointing, in the present study we did not directly test the previously described scenario. The nature of pointing produced in early stages (e.g., Brinck, 2004), and in particular the moment from which declarative pointing may reveal an intentional reading of behaviors is still being debated (e.g., D'Entremont \& Seamans, 2007) and the role of early fingertip explorations in the emergence of index-finger pointing still has to be determined.

In addition to serving different functions, pointing gestures are produced in various physical environments, which can involve one or several objects or events of interest. The comparison between obvious (A) and ambiguous (B) conditions allowed us in the present study to examine the effect of precision constraints on the features of pointing gestures. The $I M$ distance (hand shape) in imperative pointing was found to be greater in condition B than in condition A, which is consistent with our hypothesis: the shape of imperative pointing changed from whole-hand pointing to index-finger pointing with the ambiguity of the situation, namely when the target object was surrounded by distractors. By contrast, there was no significant difference in hand shape between the two conditions for declarative pointing, but this result may be explained by the very large proportion of index-finger gestures in declarative contexts, even in condition A.

The degree of arm extension did not differ between conditions $\mathrm{A}$ and $\mathrm{B}$, both for imperative and declarative pointing. The precision constraints did not influence either the body posture associated with imperative pointing, but children leaned significantly more toward the cubes in the condition $\mathrm{B}$ than in condition $\mathrm{A}$ in the declarative context. Additional studies would be needed to interpret this unexpected result. However, given that declarative pointing is mostly associated with index-finger gestures whatever the condition, we can hypothesize that a greater body contribution in this ambiguous context was an effective way for the children to stress their communicative intention. Thus, precision constraints have different effects on imperative and declarative pointing, which might be explained by initial differences in their respective features.

Finally, when distractors were around the target object, measures of hand shape for imperative pointing were similar to those reported in declarative pointing. The comparison of mean distances should not conceal for all that the existence of different individual patterns in the morphology of gestures, as the form of imperative pointing produced by some children was still close to reaching, but in a lesser proportion than in the obvious condition. Imperative pointing produced in the ambiguous condition may thus involve different types of gestures (and possibly different strategies), but the IM distance was nevertheless found to increase in the majority of children (i.e., leading to the index-finger form). It could therefore be hypothesized that imperative pointing changes as the situation becomes more ambiguous: when the target was surrounded by distractors, children indicated the precise location of the object they wanted, rather than simply requesting the object. The goal was still to obtain that object, but the nature of the gesture may have been different. However, the correlational results have shown that imperative and declarative functions remain different, as hand shape and body posture associated with imperative and declarative pointing were not correlated, whatever the precision condition (A vs. B). By contrast, the degree of arm extension was correlated between the different functions and the different conditions of pointing, suggesting that this variable reflects an individual's preference or habit rather than the influence of a specific context.

\section{Conclusions}

Although the distinction between imperative and declarative pointing gestures in young children has been widely studied, little is known regarding their respective morphological features. To our knowledge, this study is the first of its kind to describe several features of pointing using quantitative measures, thus providing a basis for future investigations. Such quantitative measures could be used to distinguish more precisely between different categories of gestures in developmental studies. Subcategories could also be defined from intermediate hand shapes or body postures, for example if the form of pointing matches neither the whole-hand gesture nor the index-finger gesture categories. However, the data obtained from our relatively small sample did not reveal any bimodal or trimodal distribution in the morphology of gestures that would have allowed us to identify such categories. 
A longitudinal study with a larger sample of children, using the same analysis program, may be especially useful to examine the development of pointing gestures from 1 to 3 years of age. In this perspective, measures of the angle between the planes defined by the index finger and the middle finger may constitute a valuable indicator of hand shape, likely to complement the measures of $I M$ distance. It may also be relevant to focus on additional variables, especially kinematic features (e.g., average speed and velocity peaks of different movements) to describe more accurately the different functions of pointing gestures and further explore the question of pointing origins.

\section{ACKNOWLEDGMENTS}

The authors thank Suzy Platcha for her precious help in collecting the data. They also thank all the children and caretakers for their participation, as well as Kévin Mare for the development of Video Analyser.

\section{FUNDING}

This research was supported by a French National Research Agency (ANR) grant, reference ANR-08-BLAN0011_01 and by a postdoctoral research grant from the Fyssen Foundation (HC).

\section{REFERENCES}

Blake, J., O'Rourke, P., \& Borzellino, G. (1994). Form and function in the development of pointing and reaching gestures. Infant Behavior and Development, 17, 195-203.

Blake, J., Vitale, G., Osborne, P., \& Olshansky, E. (2005). A crosscultural comparison of communicative gestures in human infants during the transition to language. Gesture, 5, 201-217.

Brinck, I. (2001). Attention and the evolution of intentional communication. Pragmatics \& Cognition, 9, 255-272.

Brinck, I. (2004). The pragmatics of imperative and declarative pointing. Cognitive Science Quarterly, 3, 429-446.

Bruner, J. (1978). Learning how to do things with words. In J. Bruner \& A. Garton (Eds.), Human growth and development (pp. 62-84). Oxford, England: Clarendon Press.

Bruner, J. (1983). Child's talk. New York, NY: Norton.

Butterworth, G. (2003). Pointing is the royal road to language for babies. In S. Kita (Ed.), Pointing: Where language, culture, and cognition meet (pp. 9-34). Mahwah, NJ: Erlbaum.

Butterworth, G., Franco, F., McKenzie, B., Graupner, L., \& Todd, B. (2002). Dynamic aspects of visual event perception and the production of pointing by human infants. British Journal of Developmental Psychology, 20, 1-24.

Butterworth, G., \& Morissette, P. (1996). Onset of pointing and the acquisition of language in infancy. Journal of Reproductive and Infant Psychology, 14, 219-231.

Camaioni, L. (1997). The emergence of intentional communication in ontogeny, phylogeny and pathology. European Psychologist, 2, 216-225.

Camaioni, L., Perucchini, P., Bellagamba, F., \& Colonnesi, C. (2004). The role of declarative pointing in developing a theory of mind. Infancy, 5, 291-308.

Carpendale, J. I. M., \& Carpendale, A. B. (2010). The development of pointing: From personal directedness to interpersonal direction. Human Development, 53, 110-126.
Carpenter, M., Nagell, K., \& Tomasello, M. (1998). Social cognition, joint attention, and communicative competence from 9 to 15 months of age. Monographs of the Society for Research in Child Development, 255(63), 1-143.

Cochet, H., Jover, J., \& Vauclair, J. (2011). Hand preference for pointing gestures and bimanual manipulation around the vocabulary spurt period. Journal of Experimental Child Psychology, 110, 393-407.

Cochet, H., \& Vauclair, J. (2010a). Features of spontaneous pointing gestures in toddlers. Gesture, 10, 86-107.

Cochet, H., \& Vauclair, J. (2010b). Pointing gestures produced by toddlers from 15 to 30 months: Different functions, hand shapes and laterality patterns. Infant Behavior and Development, 33, 432-442.

Colonnesi, C., Stams, G., Koster, I., \& Noom, M. J. (2010). The relation between pointing and language development: A metaanalysis. Developmental Review, 30, 352-366.

D'Entremont, B., \& Seamans, E. (2007). Do infants need social cognition to act socially? An alternative look at infant pointing. Child Development, 78, 723-728.

Esseily, R., Jacquet, A. Y., \& Fagard, J. (2011). Handedness for grasping objects and pointing and the development of language in 14-month-old infants. Laterality, 16, 565-585.

Fagard, J. (2000). Linked proximal and distal changes in the reaching behavior of 5- to 12-month-old human infants grasping objects of different sizes. Infant Behavior and Development, 23, 317-329.

Franco, F., \& Butterworth, G. (1996). Pointing and social awareness: Declaring and requesting in the second year. Journal of Child Language, 23, 307-336.

Franco, F., Perucchini, P., \& March, B. (2009). Is infant initiation of joint attention by pointing affected by type of interaction? Social Development, 18, 51-76.

Heimann, M., Strid, K., Smith, L., Tjus, T., Ulvund, S. E., \& Meltzoff, A. N. (2006). Exploring the relation between memory, gestural communication and the emergence of language in infancy: Longitudinal study. Infant and Child Development, 15, 233-249.

Iverson, J. M., \& Goldin-Meadow, S. (2005). Gesture paves the way for language development. Psychological Science, 16, 367-371.

Kaminski, T. R., Bock, C., \& Gentile, A. M. (1995). The coordination between trunk and arm motion during pointing movements. Experimental Brain Research, 106, 457-466.

Kristen, S., Sodian, B., Thoermer, C., \& Perst, H. (2011). Infants' joint attention skills predict toddlers'emerging mental state language. Developmental Psychology, 47, 1207-1219.

Leung, E., \& Rheingold, H. (1981). Development of pointing as a social gesture. Developmental Psychology, 17, 215-220.

Liszkowski, U., Carpenter, M., Henning, A., Striano, T., \& Tomasello, M. (2004). Twelve-month-olds point to share attention and interest. Developmental Science, 7, 297-307.

Liszkowski, U., Carpenter, M., \& Tomasello, M. (2008). Twelve-month-olds communicate helpfully and appropriately for knowledgeable and ignorant partners. Cognition, 108, 732-739.

Liszkowski, U., Schäfer, M., Carpenter, M., \& Tomasello, M. (2009). Prelinguistic infants, but not chimpanzees, communicate about absent entities. Psychological Science, 20, 654-660.

Liszkowski, U., \& Tomasello, M. (2011). Individual differences in social, cognitive, and morphological aspects of infant pointing. Cognitive Development, 26, 16-29.

Lock, A. J., Young, A. W., Service, V., \& Chandler, P. (1990). Some observations on the origins of the pointing gesture. In V. Volterra \& C. J. Erting (Eds.), From gesture to language in hearing and deaf children (pp. 42-55). Berlin, Germany: Springer-Verlag. 


\section{H. Cochet et al.}

Mare, K. (2010). Video Analyser: A program for the analysis of movements. Available from marianne.jover@univ-amu.fr.

Masataka, N. (2003). From index-finger extension to index-finger pointing: Ontogenesis of pointing in preverbal infants. In S. Kita (Ed.), Pointing: Where language, culture, and cognition meet (pp. 69-84). Mahwah, NJ: Erlbaum.

Matthews, D., Behne, T., Lieven, E., \& Tomasello, M. (2012). Origins of the human pointing gesture: a training study. Developmental Science, 15, 817-829.

Moll, H., \& Tomasello, M. (2007). How 14- and 18-month-olds know what others have experienced. Developmental Psychology, 43, 309-317.

Moore, C., \& Corkum, V. (1994). Social understanding at the end of the first year of life. Developmental Review, 14, 349-372.

O’Neill, D. K., \& Topolovec, J. C. (2001). Two-year-old children's sensitivity to the referential (in)efficacy of their own pointing gestures. Journal of Child Language, 28, 1-28.

Özçaliskan, S., \& Goldin-Meadow, S. (2009). When gesture-speech combinations do and do not index linguistic change. Language and Cognitive Processes, 24, 190-217.

Palmquist, C. M., \& Jaswal, V. K. (2012). Pre-schoolers expect pointers (even ignorant ones) to be knowledgeable. P sychological Science, 23, 230-231.
Rowe, M. L., \& Goldin-Meadow, S. (2009). Early gesture selectively predicts later language learning. Developmental Science, $12,182-187$.

Sacrey, L. A. R., Karl, J. M., \& Whishaw, I. Q. (2012). Development of rotational movements, hand shaping, and accuracy in advance and withdrawal for the reach-to-eat movement in human infants aged 6-12 months. Infant Behavior \& Development, 35, 543-560.

Seegelke, C., Hughes, C. M. L., \& Schack, T. (2011). An investigation into manual asymmetries in grasp behavior and kinematics during an object manipulation task. Experimental Brain Research, 215, 65-75.

Tomasello, M. (2008). Origins of human communication. Cambridge, MA: MIT Press.

Tomasello, M., \& Call, J. (1997). Primate cognition. New York, NY: Oxford University Press.

Vygotsky, L. S. (1988). Development of the higher mental functions. In K. Richardson \& S. Sheldon (Eds.), Cognitive development in adolescence (pp. 61-80). Hove, England: Erlbaum.

Received July 9, 2013

Revised January 6, 2014

Accepted January 25, 2014 